\title{
Towards a Global Germline Ethics?
}

\section{Human Heritable Genetic Modification and the Future of \\ Health Research Regulation}

\author{
Sarah Chan
}

\subsection{INTRODUCTION}

Human germline genetic modification (HGGM) has been the subject of bioethical attention for over four decades. Recently, however, two areas of biomedical technology have revived debates over HGGM. First, the development of 'mitochondrial replacement therapy' (MRT) represents, some have argued, a form of HGGM, since it affects the genetic makeup of the resulting children in a way that may be passed on to future generations. Second, the advent of genome editing ${ }^{1}$ technologies has made heritable genetic modification of humans for the first time a genuinely practicable possibility: one that was dramatically and prematurely realised when, in November 2019, it was announced that two genome-edited babies had already been born. ${ }^{2}$ Amid renewed scrutiny of human genome editing, emerging clinical uses of MRTs, and the increasing globalisation of science and of health technology markets, the question of how HGGM can and should be regulated has gained new salience. Moreover, having been so long contested and in relation to such fundamental concepts as 'human dignity' and 'human nature', the issue of germline modification has assumed a significance beyond its likely direct consequences for human health.

The current 'regulatory moment' with respect to HGGM thus perhaps represents something of a watershed for the global governance of science more generally. Further, both the potential impacts of the technology, and the moral and political power of the human genome as a metaphor through which to negotiate competing visions of human nature and society, require us to consider these issues at a global scale. This also creates an opportunity for critical exploration of novel approaches to regulation.

Following on from the previous chapter's analysis, this chapter considers broader lessons we might learn from examining the challenges of HGGM for the future of health research regulation. HGGM, I suggest, is a contemporary global regulatory experiment-in-progress through which we can re-imagine the regulation of (in particular, ethically contentious) science and innovation: what it should address, what its purposes might be, and how, therefore, we should go about shaping global scientific regulation. Through examining this, I argue that such regulation should focus on processes and practices, rather than objects; and that its utility lies

${ }^{1}$ Early discussions of these technologies often referred to 'gene editing'; in this chapter I employ the term 'genome editing', a usage that has since become more standard.

2 D. Cyranoski and H. Ledford, 'Genome-Edited Baby Claim Provokes International Outcry', (2018) Nature, 563 (7733), $607-608$. 
more in mediating these processes than in establishing absolute prohibitions or bright lines. Especially in the case of emerging and controversial technologies, regulation plays an important role in negotiating ideas of responsibility within the science-society discourse. In so doing, it also affects, and should be shaped by attention to, the global dynamics of science and the consequences for global scientific justice.

\subsection{GERMLINE TECHNOLOGIES: A BRIEF OVERVIEW}

Earlier techniques used for genetic modification were inefficient and simply impractical to allow the creation of genetically engineered humans. ${ }^{3}$ The 'game-changing' aspect of genome editing technologies ${ }^{4}$ is their ability to achieve more precise gene targeting, with much higher efficiency, in a wide range of cell types including human embryos. The best-known genome editing technology is the CRISPR/Cas9 system, the use of which was described in 2012.5 Following the publication of the CRISPR/Cas9 method, it rapidly became clear that HGGM needed urgently to be reconsidered as a real possibility. In 2015, reports of the first genome editing of human embryos ${ }^{6}$ spurred scientists to call for restrictions on the technology, ${ }^{7}$ and prompted further investigations of the associated ethical and policy issues by various national and international groups. ${ }^{8}$

Notably, many reports, including those of the US National Academies 9 and the UK's Nuffield Council on Bioethics, ${ }^{10}$ concluded that heritable human genome editing could be acceptable, providing certain conditions were met. These conditions included further research to ensure safety before proceeding to clinical application, and sufficient time for broad and inclusive engagement on governance. Neither of these conditions were fulfilled, however, when He Jiankui announced to the supposedly unsuspecting world ${ }^{11}$ that he had already attempted the procedure.

Somewhat before genome editing technologies came onto the scene, MRTs were already being developed as a treatment for certain forms of mitochondrial disease. ${ }^{12}$ According to the

3 The methods developed in the 1980 s for producing transgenic mice, for example (see B. H. Koller and O. Smithies, 'Altering Genes in Animals by Gene Targeting', [1992] Annual Review of Immunology, 10, 705-730), required extensive manipulation of embryonic stem cells (ESC) in vitro, followed by injecting these cells to form chimeric embryos, genetically screening a large number of progeny, and then selectively cross-breeding them to produce the desired genetic makeup - all steps ethically unthinkable to perform in humans.

4 H. Ledford, 'CRISPR, the Disruptor', (2015) Nature, 522(7554), 20-24.

5 M. Jinek et al., 'A Programmable Dual-RNA-Guided DNA Endonuclease in Adaptive Bacterial Immunity', (2012) Science, $337(6096), 816-821$.

6 P. Liang et al., 'CRISPR/Cas9-Mediated Gene Editing in Human Tripronuclear Zygotes', (2015) Protein Cell, 6(5), $363-372$.

7 D. Baltimore et al., 'Biotechnology. A Prudent Path Forward for Genomic Engineering and Germline Gene Modification', (2015) Science, 348(6230), 36-38; E. Lanphier et al., 'Don't Edit the Human Germ Line', (2015) Nature, 519(7544), 410-441.

8 Reviewed in C. Brokowski, 'Do CRISPR Germline Ethics Statements Cut It?', (2018) The CRISPR Journal, $1(2), 115$.

9 Committee on Human Gene Editing, Human Genome Editing: Science, Ethics and Governance (Washington, DC: N. A. Press, 2017).

${ }^{10}$ Nuffield Council on Bioethics, 'Genome Editing and Human Reproduction', (Nuffield Council on Bioethics, 2018).

${ }^{11}$ Although, it would later transpire, more than a few international academics knew of He's work prior to the announcement, provoking questions as to why the work was not flagged earlier (N. Kofler, 'Why Were Scientists Silent over Gene-Edited Babies?', (2019) Nature, 566(7745), 427).

${ }^{12}$ Mitochondria are numerous organelles within each cell that produce energy via chemical reactions and carry their own genome, separate to nuclear DNA. Some of the genes required for mitochondrial function are encoded within the nuclear DNA, while others are in the mitochondrial genome (mtDNA) itself. Since most of the cytoplasm of a developing embryo comes from the egg, mitochondria are transmitted almost exclusively from the oocyte to offspring, 
possibility foreseen in the 2008 amendments to the Human Fertilisation and Authority Act, and following an extensive consultation process, in 2015 it became legal for MRT to be licensed in the UK. ${ }^{13}$ In the USA, the Institute of Medicine likewise concluded that MRT could be acceptable, ${ }^{14}$ though it is not currently legal in the USA. Pre-empting the regulatory process, however, in September 2016 John Zhang, an American scientist, announced that he had already performed the first successful use of MRT in the clinic, ${ }^{15}$ using embryos created in the USA and shipped to Mexico for intra-uterine transfer.

While reams have been written on the ethics of HGGM, the most pressing questions with respect to HGGM no longer concern whether we ought to do it at all, but how; where; by and for whom; and with (or without) what authority it will be done. This is not a claim about the inadequacy of regulation in the face of technological inevitability but a statement of where things currently stand ethically and legally, as well as scientifically. MRT is legal and being carried out in a number of countries; heritable genome editing, while not yet legalised, has been deemed ethically acceptable in principle. One way or another, HGGM is becoming a reality; regulation can guide this process. To do so effectively will require careful consideration of what is regulated and how, with what justifications, and with whose participation.

\subsection{WHAT ARE WE REGULATING? WHAT SHOULD WE REGULATE?}

As pointed out in the previous chapter (see Isasi, Chapter 34 in this volume) regulation can serve to articulate normative concerns but does not always do so coherently or consistently. In setting out to regulate 'germline modification' or 'the human genome', what concerns might be entangled?

The term 'germline modification' is itself subject to interpretation. Technically speaking, 'the germline' can encompass any cell that is part of the germ lineage, including gametes and embryos; thus a prohibition on modifying the germline might be taken to preclude any use of genome editing in human embryos, including for basic research. Early calls for a moratorium favoured this highly restrictive approach: some argued that because 'genome editing in human embryos ... could have unpredictable effects on future generations ... scientists should agree not to modify the DNA of human reproductive cells'. ${ }^{16}$ This, however, ignores that genome editing of human embryos is only likely to have direct effects on future generations if those embryos become people! Context, in other words, is key.

Moreover, novel technologies may potentially render 'the germline' an impossibly broad category. It is now possible to reprogramme somatic cells to pluripotent cells, ${ }^{17}$ and to turn pluripotent cells into gametes. ${ }^{18}$ Any cell could therefore in theory become part of the germline, meaning any genetic modification of a somatic cell could potentially be a 'germline'

with little if any contribution from the sperm. Diseases caused by mtDNA mutations are thus 'maternally inherited', that is, passed on from mother to child.

13 The Human Fertilisation and Embryology (Mitochondrial Donation) Regulations 2015.

${ }^{14}$ National Academies of Sciences, Engineering, and Medicine, Mitochondrial Replacement Techniques: Ethical, Social, and Policy Considerations, (Washington, DC: T. N. A. Press, 2016).

15 J. Hamzelou, 'Exclusive: World's First Baby Born with New "3 Parent" Technique', (New Scientist, 27 September 2016).

${ }^{16}$ Lanphier et al., 'Don't Edit the Human Germ Line', 410.

${ }^{17}$ K. Takahashi et al., 'Induction of Pluripotent Stem Cells from Adult Human Fibroblasts by Defined Factors', (2007) Cell, 131(5), 861-872; K. Takahashi and S. Yamanaka, 'Induction of Pluripotent Stem Cells from Mouse Embryonic and Adult Fibroblast Cultures by Defined Factors', (2006) Cell, 126(4), 663-676.

18 S. Hendriks et al., 'Artificial Gametes: A Systematic Review of Biological Progress towards Clinical Application', (2015) Human Reproduction Update, 21(3), 285-296. 
modification. It is not, however, 'the germline' in the abstract, but the continuity or otherwise of particular, modified or unmodified germlines, that should be our concern.

The 'human genome' is likewise a nebulous concept: does it refer to an individual's genome, or the combined gene pool of humanity? References to the human genome as the basis of 'the fundamental unity of all members of the human family' and 'the heritage of humanity' ${ }^{19}$ seem to suggest a collective account, but it is hard to see how the 'collective genome' could be regulated. Indeed, one might argue that the human genome, in the sense of the collective gene pool of humanity, would not be altered were genome editing to be used to introduce a sequence variant into an individual human genome that already exists within the gene pool. ${ }^{20}$

Even the term 'modification' raises questions. MRT involves no change to DNA sequence, only a new combination of nuclear and mitochondrial DNA; the Institute of Medicine report, however, recommended that its use be limited to having male children only, to avoid this 'modification' being transmitted. Yet this combination of nuclear and mitochondrial DNA might also have arisen by chance rather than design, naturally rather than via MRT. The same can be said about genome editing to introduce existing genetic sequence variants. In regulating technology, we should consider whether the focus should be on outcomes, or the actions (or inactions) leading to them - and why.

The difficulty of regulating the 'germline' or the 'human genome' highlights the problem of regulating static objects rather than the dynamic relations and practices through which these objects move. In regulating a 'thing' in itself, the law tends to fix and define it, thereby rendering it inflexible and unable to evolve to match developments in technology (see McMillan, Chapter 37 in this volume). Especially in the area of biomedicine, both the pace of research and the propensity of science to discover new and often unexpected means to its ends can result in overly specific legislative provisions becoming rapidly obsolete or inapplicable.

Examples of this can be seen in previous legislative attempts to define 'the human embryo' and 'cloning'. The original Human Fertilisation and Embryology Act (1990) s1(1)(a) defined an embryo as 'a live human embryo where fertilisation is complete'. The development of somatic cell nuclear transfer technology, the process by which Dolly the sheep was cloned, immediately rendered this definition problematic, since embryos produced via this technique do not undergo fertilisation at all. Addressing this legal lacuna necessitated the hurried passage of the Human Reproductive Cloning Act 2001, ${ }^{21}$ before the eventual decision of the House of Lords brought 'Dolly'-style embryos back within the Act's purview.

A similar situation occurred in Australia, before the passage of uniform federal laws: in the state of Victoria, the embryo was defined as 'any stage of human embryonic development at and from syngamy, ${ }^{22}$ leaving unclear the status of embryos produced via nuclear transfer, in which syngamy never takes place. In South Australia, meanwhile, the law prohibited cloning, but defined 'cloning' specifically as referring to embryo splitting, again leaving nuclear transfer embryos unregulated.

These examples illustrate the pitfalls of over-determining the objects of regulation. Attempts to regulate HGGM, though, may suffer not only from being too specific in defining their objects, but also from being too vague. For example, references to 'eugenic practices' in national and

19 UNESCO, 'Universal Declaration on the Human Genome and Human Rights', (1998).

${ }^{20}$ See I. de Miguel Beriain, 'Should Human Germ Line Editing Be Allowed? Some Suggestions on the Basis of the Existing Regulatory Framework', (2019) Bioethics, 33(1), 105-111.

${ }^{21}$ D. Morgan and M. Ford, 'Cell Phoney: Human Cloning after Quintavalle', (2004) Journal of Medical Ethics, 30(6), $524-526$.

${ }^{22}$ Infertility Treatment Act 1995 (Vic), s3(1). 
international legal and policy instruments leave open the question of what actually constitutes a eugenic practice (see Isasi, Chapter 34 in this volume). Without any processes in place to determine how such terms should be interpreted, their inclusion tends to obfuscate rather than clarify the scope of regulation.

Similar examples abound: the EU Clinical Trials Directive declared: 'No gene therapy trials may be carried out which result in modifications to the subject's germ line genetic identity', ${ }^{23}$ a position further affirmed by the replacement Clinical Trials Regulation. ${ }^{24}$ But what exactly is 'germ line genetic identity'? UNESCO's Declaration opposes 'practices that could be contrary to human dignity, such as germ-line interventions', ${ }^{25}$ but does not indicate how or why germline interventions 'could be contrary to dignity' - making it difficult to distinguish whether they actually are.

The requirements of being neither too specific nor too vague may seem a Goldilocks-style demand with respect to defining the appropriate targets of regulation. What this illustrates, however, is that regulation is important for the processes and practices it establishes, as much as the definitions of objects to which these pertain.

\subsection{RESEARCH OR REPRODUCTION? THE IMPORTANCE OF CONTEXT}

In regulating HGGM, our concern should be, not whether a modification is in principle heritable but whether it is in fact inherited. The context, both social and scientific, in which the modification procedure is carried out therefore matters a great deal. Attempting to regulate this solely in terms of permitting or prohibiting particular technologies would be extremely limiting.

Instead, we should consider how our concerns can be addressed by regulating practices with respect to assisted reproduction; and relations between healthcare practitioners, healthcare systems, patients, research participants and the market. Such practices and relations are key to the processes by which future generations, and our relationships with them, are created. Regulation of this sort can be effective at transnational as well as intra-national level: crossborder surrogacy is another situation where particular practices and relations, not just the technology itself, create ethical concerns - India's regulatory response represents an example of correlative attempts to address them.

Focusing our regulatory attention on processes and relations also allows us better to distinguish desirable versus undesirable contexts for the application of technology. Basic research on embryos never destined for implantation is very different to the creation of genetically modified human beings; regulation ought accordingly to enable us clearly to separate these possibilities. This might be done in various ways, as can be seen by considering UK and USA examples.

The UK's Human Fertilisation and Embryology Act to some extent regulates embryos relationally and in terms of practices: what may be done to or with an embryo depends on the relationships among actors connected to the embryo, their relationships with the embryo itself, and the embryo's own relational context, in terms of its origin, ontology, and history. By creating the category of 'permitted embryos' as the only embryos that may be implanted, the Act effectively separates reproductive use from other applications. ${ }^{26}$

${ }^{23}$ Clinical Trials Directive, 2001/20/EC, Article 9(6).

${ }^{24}$ Clinical Trials Regulation, 536/2014.

${ }^{25}$ UNESCO, 'Universal Declaration on the Human Genome and Human Rights', (1998).

${ }^{26}$ The definition of 'permitted embryo' requires that 'no nuclear or mitochondrial DNA of any cell of the embryo has been altered' (see Human Fertilisation and Embryology Act 2008, S. 3ZA(4)(b)), which prima facie prevents 
In comparison, US federal regulation affecting HGGM incorporates aspects of both objectfocused and contextual regulation. Laws prohibiting federal funding of human embryo research apply to research with any and all embryos, regardless of origin, context, or intended destination. ${ }^{27}$ When it comes to genetically modifying those embryos, however, context matters: via the FDA's jurisdiction, current laws effectively prevent any clinical applications involving modified embryos, ${ }^{28}$ while basic research falls outside this domain.

Looking ahead to the possible futures of HGGM, what sorts of purposes and processes might we be concerned to regulate? Many of the worries that have been expressed over HGGM can be addressed via regulation (in the broad sense) of processes across different contexts. For example, the dystopian vision of a society in which parents visit a 'baby supermarket' to choose their perfect designer child is quite different to one in which the healthcare system permits parents to access reproductive interventions that have been accepted as safe (enough) for particular purposes within defined contexts. This being the case, it is far from evident that our response to these possible futures should be to forgo exploring the potential benefits of gene therapy for fear of 'designer babies': the two possibilities may be mediated via the same technologies but involve very different contexts, relationships, roles and practices. These can be differently regulated; and regulation in turn can shape which practices emerge and how they evolve.

One possible regulatory position, often motivated by a 'slippery slope' argument, is that we should prohibit all embryo genome editing research, in order to avoid the extreme dystopian futures it might one day enable. As argued above, however, context is key and focusing on technology alone fails to take account of this. Restricting research today in order to prevent one of the distant possible futures it might enable also forecloses any beneficial outcomes it might produce. To prohibit something that is prima facie acceptable merely because it may make possible the unacceptable is drawing the line in the wrong place.

Although concerns over technological development often invoke the 'slippery slope', this metaphor ignores the fact that science is not a single, uni-directional process with a defined endpoint. Instead, research and the applications it might enable are more like a 'garden of forking paths', ${ }^{29}$ a labyrinth of infinite possibilities. Regulatory slippery-slopeism, for fear of one of those possibilities, would foreclose the remainder.

That said, it might be true that what seems unacceptable from our present perspective will, from halfway down the slope, be less so. Studies of public opinions show greater acceptance of novel genetic technologies among younger demographics who have grown up in the age of IVF and genetic screening; and it was suggested with respect to MRT that this might be a slippery slope to other forms of HGGM such as genome editing ${ }^{30}$ - though this would be difficult to prove with certainty.

implantation of genetically modified embryos. MRT is rendered legal via specific provision for regulations to include within the 'permitted category' embryos that have undergone 'a prescribed process designed to prevent the transmission of serious mitochondrial disease' (see S. $3 \mathrm{ZA}(5)$ ). This provision was implemented in the Human Fertilisation and Embryology (Mitochondrial Donation) Regulations 2015.

27 Note, however, that this does not constitute a ban on embryo research across the board, only on federal funding.

${ }^{28}$ I. G. Cohen and E. Y. Adashi, 'The FDA Is Prohibited from Going Germline', (2016) Science, 353(6299), 545-546.

29 S. Chan and M.-d-J. Medina Arellano, 'Genome Editing and International Regulatory Challenges: Lessons from Mexico', (2016) Ethics, Medicine and Public Health, 2(3), 426-434; S. Chan, 'Embryo Gene Editing: Ethics and Regulation', in K. Appasani (eds), Genome Editing and Engineering: From TALENs, ZFNs and CRISPRs to Molecular Surgery (Cambridge: Cambridge University Press, 2018), pp. 454-463.

$3^{\circ}$ T. Ishii, 'Potential Impact of Human Mitochondrial Replacement on Global Policy Regarding Germline Gene Modification', (2014) Reprod Biomed Online, 29(2), 150-155; F. Baylis, 'Human Nuclear Genome Transfer (So-Called Mitochondrial Replacement): Clearing the Underbrush', (2017) Bioethics, 31(1), 7-19. 
The response to slippery-slope fears is often to try to draw a 'bright line'. Any lines we might draw, however, are liable to suffer from the above-mentioned problem of either overdetermination or vagueness. Some distinctions themselves may be less clear. For example, a commonly held position in relation to genome editing is that it should be used only for therapy, not for enhancement; but as much bioethical scholarship has revealed, the line between therapy and enhancement is not so easily defined. In a similar way, however, the definition of 'serious' disability, illness, or medical condition, for which the HFEA permits pre-implantation embryo testing, is subject to interpretation; yet its provisions have, nonetheless, been effective because there is a regulatory process for legitimate decision-making in the case of ambiguity.

Moreover, as the scientific and ethical landscape shifts, bright lines may eventually become grey areas: for example, the fourteen-day rule on embryo research was a regulatory (if not ethical) bright line for decades yet is now once again the subject of discussion(see McMillan, Chapter 37 in this volume). We should not assume that we are currently at the pinnacle of ethical understanding such that the only way is down: what we now perceive as 'slipping' might in future generations be understood as moral progress. Regulation on the slippery slope might sometimes involve drawing lines, but these should be seen as pragmatic necessities, not moral absolutes.

One supposed 'bright line' in HGGM that may not prove so clear is the somatic / germline distinction: is this really as legible or significant as it has been made out to be? Publics might not think so: recent engagement initiatives have shown widespread acceptance for therapeutically oriented genome editing, including heritable HGGM, ${ }^{31}$ while in the wake of He's attempt at creating genetically modified babies, crossing this supposed ethical Rubicon, the projected public backlash does not seem to have manifested. Moreover, in considering the possible consequences and balance of risks involved in somatic versus germline modification, we might argue that the two are not as dissimilar as might be assumed. ${ }^{22}$ Neither then in regulation should the germline be assumed to be a bright line in perpetuity: as for the fourteen-day rule, its importance as a line lies in the processes invoked when considering whether to cross it. ${ }^{33}$

\subsection{REGULATION, RESPONSIBILITY AND COOPERATIVE PRACTICE}

Given the above, what is the justification for regulating HGGM? Clearly, it is not absolute protection of the germline or genome itself: nothing stops someone visiting a plutonium refinery and exposing their germ cells to ionising radiation, or wearing too-tight underwear, and then subsequently engaging in reproduction via natural means, even though both of these processes are likely to result in heritable genetic changes. Nor would we consider it appropriate to attempt to regulate such activities. ${ }^{34}$ What, then, is regulation doing here?

${ }^{31}$ A. Van Mil et al., 'Potential Uses for Genetic Technologies: Dialogue and Engagement Research Conducted on Behalf of the Royal Society', (Hopkins van Mil, 2017).

${ }^{22}$ S. Chan, 'Playing It Safe? Precaution, Risk, and Responsibility in Human Genome Editing', (2020) Perspectives in Biology and Medicine, 63(1), 111-125.

33 McMillan, Chapter 37 in this volume; G. Cavaliere, 'A 14-Day Limit for Bioethics: The Debate over Human Embryo Research', (2017) BMC Medical Ethics, 18(1), 38; S. Chan, 'How to Rethink the Fourteen-Day Rule', (2017) Hastings Center Report, 47(3), 5-6; S. Chan, 'How and Why to Replace the 14-Day Rule', (2018) Current Stem Cell Reports, 4 (3), 228-234.

34 The principle of procreative autonomy, or reproductive liberty, is well-established ethically: see for example J. A. Robertson, Children of Choice: Freedom and the New Reproductive Technologies (Princeton University Press, 1994); R. Dworkin, Life's Dominion: An Argument about Abortion, Euthanasia and Individual Freedom (New York: Vintage, 1993). 
It may seem peculiar to allow reckless random genetic modification by individuals while the much more controlled deliberate use of directed technology should be prohibited. But the aim of regulation is not simply, or not only, to prevent certain factual outcomes. A shift in the language points us to what is at stake here: before the era of genome editing, HGGM was considered 'too risky'; now, instead, 'it would be irresponsible'.

The question then becomes what 'responsibility' requires and how should it be enacted. This highlights an important role of regulation in relation to risk, specifically in determining how we understand risk and responsibility when something goes wrong. Regulatory responsibility is not about assigning blame to individual actors, be they scientists or clinicians, but instead deciding as a society how much and what kind of risk we are collectively willing to take responsibility for. That is, in regulating to permit something, we are implicitly accepting a certain degree of accountability for the practice and for its consequences. Even as scientific responsibility has been theorised in ways that go beyond the individual scientist to the collective community, ${ }^{35}$ wider social responsibility for science requires a consideration of the interplay between social norms, regulation, and scientific practice.

Regulation therefore can also, and should, facilitate cooperative practices among different actors. At the Second International Summit on Human Genome Editing, David Baltimore described He Jiankui's attempts to create genome-edited children despite all scientific and ethical advice to the contrary as representing 'a failure of self-regulation'. ${ }^{36}$ But this is only necessarily true if we understand the primary purpose of regulation as being the absolute prevention of particular outcomes. Moreover, for 'the scientific community' to assume all of the blame for failing adequately to police its members ignores the function of states and the existence of state regulation, while arrogating what is arguably a disproportionate level of selfgovernance to scientists.

In fact, as Chinese bioethicists and legal scholars were quick to assert, ${ }^{37}$ there were various existing regulatory instruments that were breached by He's work. Genome-edited babies may have been created, but the real test of regulation is what happens next: how regulators and policy-makers (broadly categorised) respond, to this case specifically and in terms of regulating HGGM more generally, will determine whether regulation can be judged to have succeeded or failed. Notably, the imposition of a prison sentence for $\mathrm{He}^{3^{8}}$ signals that, although scientific convention and existing oversight mechanisms may not have been sufficient deterrent beforehand, the criminal law was, nonetheless, capable of administering appropriate post hoc judgment. While the criminal law in regulating science may serve a partly symbolic function in assuring social licence for morally contentious research, 39 its value in this role must be backed by a willingness to invoke its 'teeth' when needed: He’s punishment aptly demonstrates this.

35 H.-J. Ehni, 'Dual Use and the Ethical Responsibility of Scientists', (2008) Archivum Immunologiae et Therapiae Experimentalis, 56(3), 147-152; H. Jonas, The Imperative of Responsibility (Chicago University Press, 1984). Further analysis is warranted of which collective responsibilities, particularly with respect to complicity, might have been at stake in the He case.

${ }^{36}$ National Academies of Sciences, Engineering, and Medicine, 'Second International Summit on Human Genome Editing: Continuing the Global Discussion: Proceedings of a Workshop - in Brief, (Washington, DC: N. A. P. (US), 2019).

37 X. Zhai et al., 'Chinese Bioethicists Respond to the Case of He Jiankui', (Hastings Bioethics Forum, 7 February 2019), www.thehastingscenter.org/chinese-bioethicists-respond-case-jiankui/.

$3^{8}$ D. Cyranoski, 'What CRISPR-Baby Prison Sentences Mean for Research', (2020) Nature, 577(7789), 154-155.

39 M. Brazier, 'Regulating the Reproduction Business?', (1999) Medical Law Review, 7(2), 166-193; A. Alghrani and S. Chan, 'Scientists in the Dock: Criminal Law and the Regulation of Science,' in A. Alghrani et al. (eds), The Criminal Law and Bioethical Conflict: Walking the Tightrope Cambridge: Cambridge University Press, 2013), pp. 121-139. 
As this case illustrates, regulation is not just about absolute prevention, but involves mediating a complex set of relationships. Rather than viewing scientific self-regulation as a law unto itself and a separate domain, we should consider how scientists can effectively contribute to the broad project of regulation, understood as a combination of law and policy, process and practice, at multiple levels from individual to community, local to global.

\subsection{GLOBAL REGULATION AND SCIENTIFIC JUSTICE}

A common theme in discussions of the regulation of HGGM is that decisions about these technologies need to involve global participation. In order to determine what 'global participation' ought to consist of, it is worth considering why a global approach is appropriate.

Some have suggested a global approach is required because in affecting the human genome, HGGM affects all of us. George Annas and colleagues, for example, write that a decision to alter a fundamental characteristic in the definition of human should not be made ... without wide discussion among all members of the affected population ... Altering the human species is an issue that directly concerns all of us' ${ }^{4^{\circ}}$ Yet the sum of the reproductive choices being made by millions of individual humans in relation to 'natural reproduction' is vastly greater than the potential effect of what will be, in the short term at least, a tiny proportion of parents seeking to use genome editing or MRTs.

In fact, many areas of science and policy will have far-reaching consequences for humanity, some probably much more so and more immediately than HGGM. Environmental policy, for example, and the development of renewable energy sources are likely to have far greater impact on the survival and future of our species, and affect far more people now and in the future, than HGGM at the scale it is likely initially to be introduced.

Doom-laden predictions overstating the possible consequences of HGGM for 'the human race' or 'our species as a whole' tend to demand precaution, in the sense of a presumption against action, as a global approach. Such calls to action have rhetorical force and appeal to emotion, but rest on shaky premises. Overblown claims that in altering the genome we are somehow interfering with the fundamental nature of humanity are a form of genetic essentialism in themselves, implying that what makes us worthy of respect as persons and what should unite us as a moral community is nothing but base (literally!) biology.

Nevertheless, the political history of genetics has reified the moral and metaphorical power of the 'germline' concept, as something quintessential and common to all humans. This history has seen heredity, 'the germline', and 'the genome' used as a tool both for division and for unification, from eugenics to the Human Genome Project, ${ }^{41}$ imbuing genetics with a significance well beyond the mere scientific. While the biological genome as the heritage of humanity' and the basis of human dignity ${ }^{42}$ does not stand up well to analysis, the political genome, as object of multiple successive sociotechnical imaginaries, has acquired tremendous power as a regulatory fulcrum.

It is therefore genome alteration as a social and political practice, not its direct biological consequences, that we should be concerned to regulate. Beyond just requiring a global approach, this creates an opportunity to develop one. HGGM represents a socio-techno-

$4^{\circ}$ G. J. Annas et al., 'Protecting the Endangered Human: Toward an International Treaty Prohibiting Cloning and Inheritable Alterations', (2002) American Journal of Law and Medicine, 28(2-3), 151-178.

${ }^{41}$ M. Meloni, Political Biology (London: Palgrave Macmillan, 2016).

$4^{2}$ UNESCO, 'Universal Declaration on the Human Genome and Human Rights', (1998), Art. 1. 
regulatory 'event horizon', the significance of which has been contributed to by the long historical association of genetics with politics, and which aligns with a broader trend towards engaging publics in discourse over science with the aim of democratising its governance. The immediate consequences of HGGM for human reproduction are likely to be fairly small-scale, and while opening up the possibility of clinical applications of genome editing will no doubt influence the direction of the field, as MRTs are already doing for related technologies, human genome editing is just one area of the vast landscape of scientific endeavour. Yet, in providing both opportunity and momentum to produce new approaches to global regulation, HGGM may have much wider implications for the broader enterprise of science as a whole.

An important feature of any attempt to develop a global approach to regulation is that it should account for and be responsive to transnational dynamics. This requires attention to equity in terms of scientific and regulatory capacity, as well as the ability to participate in and develop ethical and social discourses over science. When it comes to emerging and contested technologies such as embryo research, cell and gene therapies, and now genome editing, countries with more advanced scientific capacity have tended also to lead in developing regulation, and to dominate ethical discussion. The resulting global regulatory 'patchwork' creates the possibility of scientific tourism, which in turn combines with uneven power over regulatory and ethical discourse to reproduce and increase global scientific inequities. This can be seen, for example, in the consequences of Zhang's Mexican MRT tourism and its effects on global scientific justice. ${ }^{43}$

Another feature of the variegated regulatory landscape for controversial technologies is concern, among countries with high scientific capacity but restrictive regulation, about remaining internationally competitive, when researchers in other countries may take advantage of lower regulatory thresholds to forge ahead. This was a prominent factor in the embryo research debates of the early 200os. Examining the expressions of concern with respect to human embryo genome editing research in China, about the science ... going forward before there's been the general consensus after deliberation that such an approach is medically warranted', ${ }^{44}$ versus in the UK being described 'an important first ... a strong precedent for allowing this type of research', 45 it seems that international dynamics and 'keeping pace' may also be a consideration here. Dominant actors may seek to control this pace to their advantage by re-asserting ethical and regulatory superiority, in the process reinforcing existing hegemonies.

The significance of the present 'regulatory moment' with respect to HGGM is that it offers opportunities to disrupt and re-evaluate these hegemonies, across geographic, cultural, disciplinary, political, and epistemic boundaries. This should include critical attention to the internalised narratives of science: in particular, the problem of characterising science as a competitive activity. The race to be first, the scientific 'cult of personality' and narratives of scientific heroism (or in the case of He Jiankui, anti-heroism) may serve to valorise and promote scientific achievement, but also drive secrecy and create incentives for 'rogue science'. What alternative narratives might we develop, to chart a better course?

43 S. Chan et al., 'Mitochondrial Replacement Techniques, Scientific Tourism, and the Global Politics of Science', (2017) Hastings Center Report, 47(5), 7-9.

44 E. Callaway, 'Second Chinese Team Reports Gene Editing in Human Embryos', (2016) Nature, doi:10.1038/ nature.2016.19718.

45 E. Callaway, 'Embryo Editing Gets Green Light', (2016) Nature, 530(7588), 18. 


\subsection{CONCLUSION: WHERE NEXT FOR GLOBAL GERMLINE REGULATION?}

Seeking a new paradigm for global health research regulation will require a conscious effort to be more inclusive. We need to examine what constitutes effective engagement in a global context and how to achieve this, across a plurality of cultural and political backgrounds, varying levels of scientific capacity and science capital, and different existing regulations.

Consider, for example, the contrasts that might emerge between the UK and China, where expectations over discourse, governance and participation differ from those in which UK public engagement has been theorised and developed. ${ }^{4}{ }^{6}$ Distinct challenges will arise for engagement in Latin America, where the politics of gender and reproduction overtly drive regulation, and where embryo research and reproductive technologies are heavily contested. The discourse is not necessarily uniform among all countries: discussing embryo genome editing is more challenging where IVF itself is still controversial. In approaching these issues, we need also to be aware of potential negative impacts the discussion may have, for example on women's access to reproductive health services.

Furthermore, we need to engage not just with publics and not just 'the scientific community' but with scientific communities. As we recognise in the field of engagement that there is not just a single unitary Public but a wide range of publics with different perspectives, values and beliefs, we need also to acknowledge pluralism of values, practices and motivations among scientists. In thinking about the governance of science, we must consider what factors might influence these and how, as an indirect form of regulating research. Some attention has already been given to the potential of actors such as journal publishers and funders to shape scientific culture and influence behaviour; further research might more clearly delineate these evolving regulatory roles, their limitations and how they work in tandem with 'harder' forms of regulation such as criminal law.

At the time of writing, the various proposed approaches to global governance of human genome editing have yet to coalesce into a single solution. The He incident triggered renewed calls for a moratorium. ${ }^{47}$ While the scientific academies behind the International Summits were already considering aspects of regulation, the process was probably likewise hastened by these events, resulting in the formation of an International Commission; the WHO have launched their own inquiry; $4^{8}$; and numerous statements have been published over the past five years, ${ }^{49}$ with many initiatives ongoing and proposals issued.

It seems clear that a moratorium is unlikely to emerge as the answer, despite reactions to He's transgression. In the first place, it is far from clear that a moratorium would have prevented He's experiment: the consensus of scientific communities publicly expressed was already that it should not be done. A moratorium without enforcement mechanisms would have been no more effective than the existing guidelines; and any symbolic value a moratorium would have would be rapidly eroded if it were not respected. Other proposals include, as per the WHO, a registry to promote greater information and transparency and facilitate the involvement of wider scientific players including funders and publishers; a global observatory ${ }^{50}$ is another proposed

$4^{6}$ J. Zhang, 'Comment: Transparency Is a Growth Industry', (2017) Nature, 545(7655), S65.

47 E. S. Lander et al., 'Adopt a Moratorium on Heritable Genome Editing', (2019) Nature, 567(7747), 165-168.

$4^{8}$ World Health Organisation, 'Global Health Ethics: Human Genome Editing', www.who.int/ethics/topics/humangenome-editing/en/.

49 Brokowski, 'Do CRISPR Germline Ethics Statements Cut It?'.

$5^{\circ}$ J. Benjamin Hurlbut et al., 'Building Capacity for a Global Genome Editing Observatory: Conceptual Challenges', (2018) Trends in Biotechnology, 36(7), 639-641; S. Jasanoff et al., 'Democratic Governance of Human Germline Genome Editing', (2019) The CRISPR Journal, 2(5), 266-271; K. Saha et al., 'Building Capacity for a Global Genome Editing Observatory: Institutional Design', (2018) Trends in Biotechnology, 36(8), 741-743. 
mechanism to enable governance. With any of these, we will still need to attend to the dynamics of discourse, which interests are represented and how.

With that in mind, perhaps a single solution is not what we should be seeking. The proliferation of initiatives aimed at determining principles and frameworks for acceptable governance of HGGM may lead some to wonder whether we really need so many cooks for this broth, and to raise objections regarding potential inconsistencies when multiple bodies are charged with a similar task. Yet, even among the number of bodies that currently exist, the full range of diverse views has not been represented. The existence of multiple parallel discourses is not necessarily a bad thing: more can be better if it allows for broader representation. The meta-solution of integrating these is the challenge; approaching regulation as dynamic, constituted by practices and concerned with processes and relations, may be a way to meet it. 